\title{
The Effects of Ba0.85Ca0.15Zr0.1Ti0.903 Addition on the Phase, Microstructure, and Thermoelectric Properties of Ca3Co409 Ceramics
}

Panupong JAIBAN ( $\sim$ panupongj@kmutnb.ac.th )

King Mongkut's University of Technology North Bangkok https://orcid.org/0000-0002-7660-1094 Pimpilai WANNASUT

Chiang Mai University

Anucha WATCHARAPASORN

Chiang Mai University

\section{Research Article}

Keywords: Thermoelectric-ferroelectric, Chemical defects, Ca-based oxide thermoelectric, BCZT doping, Ptype oxide thermoelectric

Posted Date: December 6th, 2021

DOI: https://doi.org/10.21203/rs.3.rs-1130828/v1

License: (9) This work is licensed under a Creative Commons Attribution 4.0 International License. Read Full License

Version of Record: A version of this preprint was published at Journal of Asian Ceramic Societies on March 13th, 2022. See the published version at https://doi.org/10.1080/21870764.2022.2048441. 
The effects of $\mathrm{Ba}_{0.85} \mathrm{Ca}_{0.15} \mathrm{Zr}_{0.1} \mathrm{Ti}_{0.9} \mathrm{O}_{3}$ addition on the phase, microstructure, and thermoelectric properties of $\mathrm{Ca}_{3} \mathrm{Co}_{4} \mathrm{O}_{9}$ ceramics

\author{
Panupong JAIBAN $^{\mathrm{a}, \mathrm{b}}{ }^{\mathrm{b}}$, Pimpilai WANNASUT ${ }^{\mathrm{c}}$, Anucha WATCHARAPASORN $^{\mathrm{b}, \mathrm{c}, \mathrm{d}}$ \\ ${ }^{a}$ Faculty of Science, Energy and Environment, King Mongkut's University of \\ Technology North Bangkok, Rayong Campus, Rayong 21120, Thailand \\ ${ }^{\mathrm{b}}$ Research Center for Quantum Technology, Faculty of Science, Chiang Mai University,
} Chiang Mai, 50200, Thailand

${ }^{\mathrm{c}}$ Department of Physics and Materials Science, Faculty of Science, Chiang Mai University, Chiang Mai 50200, Thailand

${ }^{\mathrm{d}}$ Center of Excellence in Materials Science and Technology, Materials Science Research Center, Faculty of Science, Chiang Mai University, Chiang Mai 50200, Thailand

* Corresponding author. Tel: +66 38627000 ext. 5710.

E-mail address: panupongj@kmutnb.ac.th

\begin{abstract}
In this work, the influences of $\mathrm{Ba}_{0.85} \mathrm{Ca}_{0.15} \mathrm{Zr}_{0.1} \mathrm{Ti}_{0.9} \mathrm{O}_{3}$ (BCZT) addition on phase, microstructure, and thermoelectric properties of $\mathrm{Ca}_{3} \mathrm{Co}_{4} \mathrm{O}_{9}(\mathrm{CCO})$ were investigated. $(1-x)$ CCO- $(x)$ BCZT ceramics where $x=0,0.003,0.005$, and 0.010 were fabricated successfully via a conventional solid-state sintering at $1,223 \mathrm{~K}$ for $24 \mathrm{hrs}$. The substitution of BCZT introduced the chemical defects $\left(V_{C o}^{\prime \prime}, V_{C o}^{\prime \prime \prime}, V_{C a}^{\prime \prime}\right)$ in CCO ceramic, which increased charge carrier concentration and enhanced the electrical conductivity. The presence of $\mathrm{Co}^{3+}$ improved the Seebeck coefficients of CCO ceramic. The thermal conductivity of CCO ceramic decreased when BCZT was added. The addition of BCZT
\end{abstract}


at $x=0.010$ promoted the highest thermoelectric power factor $\left(\mathrm{PF} \sim 235 \mu \mathrm{W} / \mathrm{mK}^{2}\right)$, and the highest figure of merit $(Z T \sim 0.5)$ at $800 \mathrm{~K}$, which present this ceramic an alternative p-type oxide thermoelectric for a high-temperature thermoelectric device.

Keywords: Thermoelectric-ferroelectric; Chemical defects; Ca-based oxide thermoelectric, BCZT doping, P-type oxide thermoelectric

\section{Introduction}

Thermoelectric material can convert waste heat to electricity directly, which causes the material to become a candidate for a green energy harvester. For practical application, a figure of merit $\left(Z T=\sigma \alpha^{2} T / \kappa\right)$ equal to 1 of thermoelectric material is typically required [1]. $Z T$ can be calculated from electrical conductivity $(\sigma)$, Seebeck coefficients $(\alpha)$, thermal conductivity $(\kappa)$, and absolute temperature $(T)$. For example, the intermetallic compounds: $\mathrm{Bi}_{2} \mathrm{Te}_{3}$ and $\mathrm{PbTe}$ exhibit high thermoelectric performance and $Z T$ close to 1 . However, both materials are highly oxidative and toxic [2], which several researchers are avoiding this drawback. For this reason, the metal oxide thermoelectric ceramics are extensively focused because of their good thermoelectric properties and high heat resistance [2].

Among oxide thermoelectric materials, $\mathrm{Ca}_{3} \mathrm{Co}_{4} \mathrm{O}_{9}$ (CCO) misfit-layered compound is one of the attractive materials, particularly the CCO single crystal has shown high $Z T(\sim 0.87$ at $973 \mathrm{~K})$ [3]. Nevertheless, it is pretty expensive and difficult to prepare on a large scale. The polycrystalline ceramic of CCO is therefore pointed out, although it still has lower $Z T$. There are many attempts to improve $Z T$ of $\mathrm{CCO}$ by doping at Ca-site or Co-site [4-6]. The primary enhancement of ZT of CCO ceramics 
resulted from increased $\alpha, \sigma$, or decrease in $\kappa$. From this aspect, we are interested in the improvement in thermoelectric properties of $\mathrm{CCO}$ by doping.

$\mathrm{Ba}_{0.85} \mathrm{Ca}_{0.15} \mathrm{Zr}_{0.1} \mathrm{Ti}_{0.9} \mathrm{O}_{3}$ (BCZT) is a lead-free ferroelectric material, which has exhibited a potential performance to replace PZT material in several electronic devices such as sensors, actuators, and capacitors [7] due to its suitable dielectric and piezoelectric properties. BCZT is quite sensitive with deviation from its stoichiometry and ion doping, which produced phase transition, crystal structure, and chemical defects $[8,9]$. The generated defects can dope free mobile charge carriers and enhance electronic and thermal transport in BCZT material [9]. This contribution is expected to influence the thermoelectric properties of CCO ceramic. Hence, the addition of BCZT on $\mathrm{CCO}$ ceramic becomes a challenge for the thermoelectric investigation of $\mathrm{CCO}$ based material.

Nowadays, the thermoelectric properties of CCO ceramic, which BCZT dopes, have not been reported yet. Thus, in this work, we then presented the effects of BCZT on the thermoelectric properties of CCO ceramics. $(1-x) \mathrm{CCO}-(x) \mathrm{BCZT}$ ceramics where $x=0,0.003,0.005$, and 0.010 weight fraction were fabricated via solid-state sintering. Scanning electron microscopy (SEM) and X-ray diffraction (XRD) techniques were used to determine the phase of all ceramics. X-ray absorption spectroscopy (XAS) was employed to survey the oxidation state of elements in these ceramics. The influences of chemical defects, charge concentration, and oxidation state on thermoelectric properties of the doped-CCO ceramics would discuss in detail. 


\section{Material and methods}

\subsection{Materials}

The starting materials were $\mathrm{CaCO}_{3}\left(99.5 \%\right.$, Riedel-de Haën) and $\mathrm{Co}_{3} \mathrm{O}_{4}(99.5 \%$, Aldrich) for $\mathrm{CCO}, \mathrm{BaCO}_{3}$ (98.5\%, Sigma-Aldrich), $\mathrm{CaCO}_{3}$ (99.95\%, Sigma-Aldrich), $\mathrm{TiO}_{2}$ (99.9\%, Riedel-de Haën), and $\mathrm{ZrO}_{2}$ (98.5\%, Sigma-Aldrich) for BCZT.

\subsection{Preparation of $(1-x) \mathrm{CCO}-(x) \mathrm{BCZT}$ powders}

The starting oxides were weighed following the chemical formula of $\mathrm{Ca}_{3} \mathrm{Co}_{4} \mathrm{O}_{9}$ (CCO) and $\left(\mathrm{Ba}_{0.85} \mathrm{Ca}_{0.15}\right) \mathrm{Zr}_{0.1} \mathrm{Ti}_{0.9} \mathrm{O}_{3}$ (BCZT). The oxide mixtures were ball milled in ethanol ( $>99.5 \%$, Merck) for 24 hrs by using a conventional mixed-oxide method. The mixed powders were dried at $393 \mathrm{~K}$ for $24 \mathrm{hrs}$ and calcined in an alumina crucible at $1,023 \mathrm{~K}$ for $24 \mathrm{hrs}(\mathrm{CCO})$ and at $1,373 \mathrm{~K}$ for $3 \mathrm{hrs}$ (BCZT). After sieving, (1-x)CCO(x)BCZT binary powders where $x=0,0.003,0.005$, and 0.010 weight fraction were mixed again in ethanol (>99.5\%, Merck) for $6 \mathrm{hrs}$ and dried at $393 \mathrm{~K}$ for $24 \mathrm{hrs}$.

\subsection{Preparation of (1-x)CCO- $(x) B C Z T$ ceramics}

A conventional solid-state sintering technique was used to fabricate all samples. After obtaining $(1-x) \mathrm{CCO}-(x) \mathrm{BCZT}$ powders, one drop of $3 \mathrm{wt} \%$ PVA (polyvinyl alcohol, Merck) binder was added to the $(1-x)$ CCO- $(x)$ BCZT powders $(\sim 1 \mathrm{~g})$. The mixed powders were pressed into pellets with a diameter of $10 \mathrm{~mm}$ and thickness of 2 mm using a uniaxial press with $187.3 \mathrm{MPa}$. Binder removal was carried out by heating the pellets at $773 \mathrm{~K}$ for $1 \mathrm{hr}$ in an air atmosphere with a heating/cooling rate of $5 \mathrm{~K} / \mathrm{min}$. These pellets were then sintered at $1,223 \mathrm{~K}, 1,273 \mathrm{~K}, 1,323 \mathrm{~K}$, and 1,373 K for $24 \mathrm{hrs}$ of 
dwell time in an air atmosphere with a heating/cooling rate of $5 \mathrm{~K} / \mathrm{min}$ on an alumina plate.

\subsection{Characterization}

The bulk densities of all ceramics were determined using the Archimedes' method. Then, the relative densities of the ceramics were calculated from the relationship between theoretical and bulk densities. In this study, the theoretical density

of CCO was $4.68 \mathrm{~g} / \mathrm{cm}^{3}$ [10]. The microstructural observation of the samples was carried out employing SEM (JEOL JSM 6335F). Phase identification of the sintered samples was investigated in a 2-theta range of $20-70^{\circ}$ using XRD (Miniflex, Rigaku). The chemical composition of the ceramics was studied employing X-ray absorption near edge structure (XANES) at beamline 5.2:SUT-NANOTEC-SLRI XAS, Synchrotron Light Research Institute (SLRI), Thailand.

\subsection{Thermoelectric properties}

The thermoelectric properties from $300 \mathrm{~K}$ to $800 \mathrm{~K}$ were investigated. The electrical conductivity and the Seebeck coefficients were measured using an instrument designed by Iwanaga et al. [11]. The charge carrier concentration of the samples was investigated by Hall measurement. The laser flash instrument (Netzsch, LFA 457) was employed for measuring thermal conductivity.

\section{Results and discussion}

\subsection{The optimized sintering temperatures and microstructural observation}

The $(1-x)$ CCO- $(x)$ BCZT ceramics with $x=0,0.003,0.005$, and 0.010 were sintered at temperature range between $1,223 \mathrm{~K}$ to $1,373 \mathrm{~K}$ for $24 \mathrm{hrs}$. The sintering 
temperatures above $1,223 \mathrm{~K}$ produced the melted and deformed pellets, which were quite difficult for studying in the phase, chemical environment, and thermoelectric properties. Thus, the pellets sintered at 1,223 $\mathrm{K}$ become representative for all ceramics. Figure 1 shows the microstructure of all ceramics. CCO ceramic had a porous microstructure, accompanied by its lowest relative density $(\sim 90 \%)$, as shown in Table 1. When BCZT was added, the microstructure of CCO was improved. The relative densities of the doped ceramics, which are listed in Table 1, increased gradually with increasing BCZT content, and the maximum value was about $98 \%$ for $x=0.010$.

Moreover, the SEM images revealed the presence of other phases together with CCO. It should be noted that the observed phase was much and too small. It was quite tricky for obvious phase identification by energy dispersive spectroscopy (EDS). However, we could use EDS to identify the phase of $\mathrm{CaO}$ (white color) at $x=0.005$. The interpretation of different phases would be discussed together with XRD results in the next section.

\subsection{Phase identification}

The XRD patterns of all ceramics are illustrated in Fig. 2. The composition $x=0$ showed the single phase of CCO, which is related to the standard card (PDF 21-0139). XRD patterns of the doped CCO ceramics with $x \leq 0.005$ were not different from that of CCO ceramic. However, from their SEM images, the results confirmed the presence of other phases. The content of these phases might below, which could not be observed by XRD patterns. When BCZT content was added to $x=0.010$, the XRD pattern could reveal additional peaks of other phases. The concentration of these phases might have been much (see in Fig. 1 at $x=0.010$ ); thus, XRD analysis could detect it. These phases 
were $\mathrm{Ca}_{3} \mathrm{Co}_{2} \mathrm{O}_{6}, \mathrm{CoO}, \mathrm{Co}_{3} \mathrm{O}_{4}$, and $\mathrm{CaO}$, generated by variation in the ratio of $\mathrm{Ca} / \mathrm{Co}$ suggested by Tahashi et al. [12]. The general $\mathrm{Ca} / \mathrm{Co}$ ratio of single-phase $\mathrm{CCO}$ is $3 / 4$. The addition of $\mathrm{BCZT}$ might increase the $\mathrm{Ca} / \mathrm{Co}$ ratio to $3 / 2$ due to $\mathrm{BCZT}$ was composed of $\mathrm{Ca}$ ions. This therefore induced the partial decomposition of $\mathrm{Ca}_{3} \mathrm{Co}_{4} \mathrm{O}_{9}$ to $\mathrm{Ca}_{3} \mathrm{Co}_{2} \mathrm{O}_{6}$ and other oxide phases [12]. The formation of these phases significantly influenced the electrical and thermal conductivity of these samples, which would be discussed later.

\subsection{Defect chemistry}

According to XRD results, the additional phases were $\mathrm{Ca}_{3} \mathrm{Co}_{2} \mathrm{O}_{6}, \mathrm{CoO}, \mathrm{Co}_{3} \mathrm{O}_{4}$, and $\mathrm{CaO}$. The creation of these phases was attributed to the $\mathrm{Ca}_{3} \mathrm{Co}_{4} \mathrm{O}_{9}$ phase transformation, which a chemical reaction in Eqn (1) could explain,

$$
\mathrm{Ca}_{3} \mathrm{Co}_{4} \mathrm{O}_{9} \rightarrow 1 / 2 \mathrm{Ca}_{3} \mathrm{Co}_{2} \mathrm{O}_{6}+3 / 2 \mathrm{CoO}+1 / 2 \mathrm{Co}_{3} \mathrm{O}_{4}+3 / 2 \mathrm{CaO}+1 / 2 \mathrm{O}_{2}
$$

$\mathrm{Ca}_{3} \mathrm{Co}_{2} \mathrm{O}_{6}$ could occur in the fluctuating stoichiometry of $\mathrm{Ca}_{3} \mathrm{Co}_{4} \mathrm{O}_{9}$, and this phase is still regarded as the thermoelectric phase. On the contrary, other oxides were in the non-thermoelectric phase and could produce chemical defects. It was worth mentioning the defect formation for each oxide. In the case of $\mathrm{CoO}$, the vacancy defects of $\mathrm{Co}^{2+}$ ions $\left(V_{C o}^{\prime \prime}\right)$ could be produced and explained by Kröger-Vink notation in Eqn.

$$
\mathrm{Co}_{\mathrm{Co}}^{x}+1 / 2 \mathrm{O}_{2}+2 e^{\prime} \rightarrow \mathrm{CoO}+V_{\mathrm{Co}}^{\prime \prime}
$$

In the case of $\mathrm{Co}_{3} \mathrm{O}_{4}$, it might have two types of cobalt vacancies. They related to tetrahedral and octahedral coordination geometries of $\mathrm{Co}^{2+}$ and $\mathrm{Co}^{3+}$ ions, respectively. 
Equations (3) and (4) present two possible vacancies of $\mathrm{Co}^{2+}$ and $\mathrm{Co}^{3+}$ ions $\left(V_{C o}^{\prime \prime \prime}\right)$, respectively.

$$
6 \mathrm{Co}_{\mathrm{Co}}^{x}+4 \mathrm{O}_{2}+12 e^{\prime} \rightarrow 2 \mathrm{Co}_{3} \mathrm{O}_{4}+6 V_{C o}^{\prime \prime}
$$

$$
3 \mathrm{Co}_{\mathrm{Co}}^{x}+2 \mathrm{O}_{2}+9 e^{\prime} \rightarrow \mathrm{Co}_{3} \mathrm{O}_{4}+3 \mathrm{~V}_{\mathrm{Co}}^{\prime \prime \prime}
$$

For $\mathrm{CaO}$, the vacancy defects of $\mathrm{Ca}^{2+}$ ions $\left(V_{C a}^{\prime \prime}\right)$ could be produced and its Kröger-Vink notation is given in Eqn. (5) [9]

$$
\mathrm{Ca}_{\mathrm{Ca}}^{x}+1 / 2 \mathrm{O}_{2}+2 e^{\prime} \rightarrow \mathrm{CaO}+V_{\mathrm{Ca}}^{\prime \prime}
$$

As seen in the equations, the creation of the cation vacancies, i.e., $V_{C o}^{\prime \prime}, V_{C o}^{\prime \prime \prime}$, and $V_{C a}^{\prime \prime}$ also produced excess free electrons. Both the cation vacancies and free electrons were regarded as mobile charge carriers, leading to doping of charge carriers in the ceramics.

\subsection{Electrical conductivity and charge carrier concentration}

Electrical conductivities $(\sigma)$ as a function of temperature range from $300 \mathrm{~K}$ to $800 \mathrm{~K}$ for all ceramics are shown in Fig. 3(a). At $300 \mathrm{~K}$, the CCO ceramics doped BCZT content $x \leq 0.005$ had electrical values nearby pure ceramic $\left(\sim 0.062 \times 10^{3} \mathrm{~S} / \mathrm{m}\right)$. Interestingly, the addition of BCZT at $x=0.010$ become effective, and the electrical conductivity values of this ceramic were enhanced greatly (from $0.062 \times 10^{3} \mathrm{~S} / \mathrm{m}$ to $\left.0.52 \times 10^{3} \mathrm{~S} / \mathrm{m}\right)$. This electrical enhancement was attributed to the increment of charge carrier concentration $(n)$ in the ceramic. The measured charge concentration at $300 \mathrm{~K}$ for the $x=0.010$ was $3.15 \times 10^{18} 1 / \mathrm{cm}^{3}$, which much higher than the value of $x=0$ 
$\left(1.31 \times 10^{15} 1 / \mathrm{cm}^{3}\right)$. The increase in charge concentration is associated with the formation of chemical defects in this ceramic. Upon increasing the temperature, electrical values of all ceramics increased because of the stimulation of thermal energy. The composition at $x=0.010$ promoted the great increase of $\sigma$ values compared with other ceramics. The feature of this trend is accompanied well by the increase of mobile charge carrier concentration of $x=0.010$, as shown in Fig. 2(b). The $n$ values at $800 \mathrm{~K}$ of the ceramics with $x=0$, and 0.010 were $1.79 \times 10^{17} 1 / \mathrm{cm}^{3}$ and $1.3 \times 10^{21} 1 / \mathrm{cm}^{3}$, respectively. In general, the increase of the mobile charge carriers at high temperatures is due to thermal activation. Eqn. 6 [4] was therefore used to investigate the activation energy $\left(E_{a}\right)$ of all ceramics,

$$
\sigma=n e a^{2}(A / T) \exp \left(-E_{a} / k_{B} T\right)
$$

where $n$ is the carrier concentration, $e$ is the charge value of electron, and $a$ is the intersite distance of hopping, $A$ is the pre-exponential term, $k_{\mathrm{B}}$ is the Boltzmann's constant, and $T$ is the absolute temperature. The plots of $\ln \sigma$ as a function of 1000/T for all ceramics are present in Fig. 2(c). $E_{a}$ can be extracted from the slope of linear fitting, and the values of all ceramic are listed in Table $1 . E_{a}$ values decreased gradually and became much lower at $x=0.010(\sim 0.059 \mathrm{eV})$. The sample with low $E_{a}$ could be stimulated easier than the sample with high $E_{a}$. Thus, this result confirmed well the electrical enhancement of BCZT-doped CCO ceramic at $x=0.010$.

\subsection{Seebeck coefficients and oxidation state}


The temperature dependence of the Seebeck coefficients $(\alpha)$ of all ceramics is illustrated in Fig. 4(a). All ceramics showed positive values of $\alpha$, indicating p-type semiconducting behavior. The ceramics with $x \leq 0.005$ had a slight increase of $\alpha$ values with increasing temperature, meanwhile, the composition $x=0.010$ had a better increase, particularly, the value become much higher at $800 \mathrm{~K}(\sim 400 \mu \mathrm{V} / \mathrm{K})$. Generally, Seebeck coefficients of the CCO misfit-layered materials are correlated with the electron system strongly [13]. Thus, $\alpha$ can be investigated using the modified Heikes formula [14]:

$$
\alpha=-\frac{k_{B}}{e} \ln \left(\frac{g_{3}}{g_{4}} \frac{1}{1-y}\right)
$$

where $g_{3}$ and $g_{4}$ are the number of configurations of $\mathrm{Co}^{3+}$ and $\mathrm{Co}^{4+}$ ions, respectively. The $y$ is the fraction of $\mathrm{Co}^{4+}$ in the $\mathrm{CoO}_{2}$ layer. Eqn. (7) suggests that $\alpha$ value can be increased by increasing $\mathrm{Co}^{3+}$ ions, which is attributed to a spin-entropy enhancement.

Fig. 4(b) shows XAS $K$-edges spectra of Co ions of all ceramics. The features at $\mathrm{A}$ and $\mathrm{B}$ peaks are associated with the transition from the Cols core level to the Co3d$\mathrm{O} 2 p$ hybridized final state and the Cols core level to the unoccupied $4 p$-derived orbitals, respectively [15]. Regarding to B peak, the maximum absorption positions shifted from $7726 \mathrm{eV}$ for $x=0$ to $7729 \mathrm{eV}$ for $x=0.010$. In general, the peak at $7726 \mathrm{eV}$ reflects the presence of $\mathrm{Co}^{2+}$; meanwhile, the peak at $7729 \mathrm{eV}$ reflects the presence of $\mathrm{Co}^{3+}$. These results supported further increase of $\mathrm{Co}^{3+}$ for the ceramic $x=0.010$ and led to its Seebeck coefficient enhancement. 


\subsection{Thermal conductivity, power factor, and figure of merit}

The temperature dependence of the total thermal conductivity $\left(\kappa_{\text {total }}\right)$ for all ceramics is shown in Fig. 5(a). The $\kappa_{\text {total }}$ values of the ceramics with $x \leq 0.005$ decreased with increasing temperature; meanwhile, the composition $x=0.010$ almost had stable values upon increasing temperature. The thermal conductivity of the composition $x=0.010$ was lowest about $0.5 \mathrm{~W} / \mathrm{mK}$ at $300 \mathrm{~K}$ and $0.4 \mathrm{~W} / \mathrm{mK}$ at $800 \mathrm{~K}$. In general, $\kappa_{\text {total }}$ in the materials can be expressed as $\kappa_{\text {total }}=\kappa_{\mathrm{ph}}+\kappa_{\mathrm{e}}$, where $\kappa_{\mathrm{ph}}$ and $\kappa_{\mathrm{e}}$ are a contribution of lattice vibration and electronic thermal conductivity, respectively. In the case of $\kappa_{\mathrm{e}}$, the value could be estimated using Wiedemann-Franz's law, which could be written as

$$
\kappa_{e}=L \sigma T
$$

Where $L$ is the Lorenz number $\left(2.45 \times 10^{-8} \mathrm{~W} \Omega \mathrm{K}^{-2}\right)$. The $\kappa_{\mathrm{ph}}$ and $\kappa_{\mathrm{e}}$ functions of a temperature of all ceramics are plotted in Fig. 5(b). It was seen that the main contribution of thermal conductivity of these ceramics resulted from $\kappa_{\mathrm{ph}}$. The decrease in $\kappa_{\mathrm{ph}}$, upon temperature increase, was attributed to phonon-phonon solid interaction at high temperature [5]. Conversely, with $\kappa_{\mathrm{ph}}$, all ceramics showed a rise of $\kappa_{\mathrm{e}}$ with increasing temperature, particularly the composition $x=0.010$. This result reflected that mobile charge carriers, i.e., cation vacancies and free electrons, not only participated in electrical conductivity but also governed the thermal conductivity of this ceramic.

The thermoelectric power factors (PF) can be calculated from $\sigma \times \alpha^{2}$, and the calculated values as a function of a temperature for all ceramics are shown in Fig. 5(c). With these ceramics, the electrical conductivity was enhanced by increased charge 
carrier concentration, and the improvement of Seebeck coefficients was attributed to the improved spin-entropy effect. The highest value of PF was about $235 \mu \mathrm{W} / \mathrm{mK}^{2}$ at $800 \mathrm{~K}$ for the composition $x=0.010$, which was more significant than CCO ceramics. Fig. 5(d) shows the figure of merit $(Z T)$ values as a function of a temperature for all ceramics. The highest value was found to be $\sim 0.5$ at $800 \mathrm{~K}$ for the composition $x=$ 0.010 .

With these results, we found that the addition of BCZT could enhance the electrical conductivity, Seebeck coefficients, and thermal conductivity of CCO ceramic by forming chemical defects. However, the doping at $x \leq 0.005$ did not promote the practical improvement of thermoelectric properties. The results here suggested the addition of BCZT at $x=0.010$ could promote a high $Z T$ value at $800 \mathrm{~K}$ of $\mathrm{CCO}$ ceramic, which may be an alternative $p$-type oxide thermoelectric material for hightemperature thermoelectric devices.

\section{Conclusions}

$(1-x)$ CCO- $(x)$ BCZT ceramics where $x=0,0.003,0.005$, and 0.010 were fabricated successfully via solid-state sintering at $1,223 \mathrm{~K}$ for $24 \mathrm{hrs}$. The addition of BCZT introduced the chemical defects in $\mathrm{CCO}$ ceramic, which increased charge carrier concentration. This contribution increased the electrical conductivity of the ceramics, particularly at $x=0.010$. The Seebeck coefficients were enhanced by presence of $\mathrm{Co}^{3+}$. The thermal conductivity of these ceramics was mainly attributed to the phonon vibration, and the lowest value was found at composition $x=0.010$. The $\mathrm{CCO}$ ceramic doped BCZT at $x=0.010$ promoted the highest values of thermoelectric power factor 
$\left(235 \mu \mathrm{W} / \mathrm{mK}^{2}\right)$ and figure of merit $(0.5)$ at $800 \mathrm{~K}$, which might find the applications for high-temperature thermoelectric devices.

\section{Acknowledgments}

This research was supported by the Program Management Unit for Human Resources \& Institutional Development, Research and Innovation [grant number B05F630113]. P. Jaiban also would like to thank partial funding support from King Mongkut's University of Technology North Bangkok. Contract no. KMUTNB-64KNOW-14.

\section{References}

[1] Tritt TM, Subramanian MA. Thermoelectric materials, phenomena, and application: A bird's eye view. MRS Bull 2006, 31: 188-198.

[2] Koumoto K, Wang YF, Zhang RZ, et al. Oxide thermoelectric materials: A nanostructuring approach. Annu Rev Mater Res 2010, 40: 363-394.

[3] Shikano M, Funahashi R. Electrical and thermal properties of single-crystalline $\left(\mathrm{Ca}_{2} \mathrm{CoO}_{3}\right)_{0.7} \mathrm{CoO}_{2}$ with a $\mathrm{Ca}_{3} \mathrm{Co}_{4} \mathrm{O}_{9}$ structure. Appl Phys Lett 2003, 82: 18511853.

[4] Liu HQ, Song Y, Zhang SN, et al. Thermoelectric properties of $\mathrm{Ca}_{3-x} \mathrm{Y}_{x} \mathrm{Co}_{4} \mathrm{O}_{9+\delta}$ ceramics. J Phys Chem Solids 2009, 70: 600-603.

[5] Nong NV, Liu CJ, Ohtaki M, et al. Improvement on the high temperature thermoelectric performance of Ga-doped misfit-layered $\mathrm{Ca}_{3} \mathrm{Co}_{4-x} \mathrm{Ga}_{x} \mathrm{O}_{9+\delta}(x=0$, 0.05, 0.1, and 0.2). J Alloys Comp 2010, 491: 53-56.

[6] Xu LX, Li F, Wang Y, et al. High-temperature transport and thermoelectric properties of $\mathrm{Ca}_{3} \mathrm{Co}_{4-x} \mathrm{Ti}_{x} \mathrm{O}_{9}$. J Alloys Comp 2010, 501: 115-119. 
[7] Liu W, Ren X, Large piezoelectric effect in Pb-free ceramics. Phys Rev Lett 2009, 103: 257602.

[8] Jaiban $\mathrm{P}$, Tongtham $\mathrm{M}$, Wannasut $\mathrm{P}$, et al. Phase characteristics, microstructure, and electrical properties of $(1-x) \mathrm{BaZr}_{0.2} \mathrm{Ti}_{0.8} \mathrm{O}_{3^{-}}$ $(x)\left(\mathrm{Ba}_{0.7} \mathrm{Ca}_{0.3}\right)_{0.985} \mathrm{La}_{0.01} \mathrm{TiO}_{3}$ ceramics. Ceram Int 2019, 45: 17502-17511.

[9] Jaiban P, Tongtham M, Wannasut P, et al. Dielectric response on ultraviolet light irradiation of $\mathrm{Ba}_{0.85} \mathrm{Ca}_{0.15} \mathrm{Zr}_{0.1} \mathrm{Ti}_{0.9} \mathrm{O}_{3}$ based ceramics. Mater Lett 2019, 243: $169-172$.

[10] Madre MA, Costa FM, Ferreira NM, et al. Preparation of high-performance $\mathrm{Ca}_{3} \mathrm{Co}_{4} \mathrm{O}_{9}$ thermoelectric ceramics produced by a new two-step method. J Eur Ceram Soc 2013. 33: 1747-1754.

[11] Iwanaga S, Toberer ES, Londe AL, et al. A high temperature apparatus for measurement of the Seebeck coefficient. Rev Sci Instrum 2011, 82: 063905.

[12] Tahashi M, Tanimoto T, Goto H, et al. Sintering temperature dependence of thermoelectric performance and crytal phase of calcium cobalt oxides. J Am Ceram Soc 2010, 93: 3046-3048.

[13] Limelette P, Hardy V, Auban-Senzier P, et al. Strongly correlated properties of the thermoelectric cobalt oxide $\mathrm{Ca}_{3} \mathrm{Co}_{4} \mathrm{O}_{9}$. Phys Rev B 2005, 71: 233108.

[14] Koshibae W, Tsutsui K, Maekawa S. Thermopower in cobalt oxides. Phys Rev B 2000, 62: 6869-6872.

[15] Chen JL, Liu YS, Liu CJ, et al. Effect of Mn doping on the physical properties of misfit-layered $\mathrm{Ca}_{3} \mathrm{Co}_{4} \mathrm{O}_{9+\delta}$. J Phys D Appl Phys 2009, 42: 135418. 
Fig.1

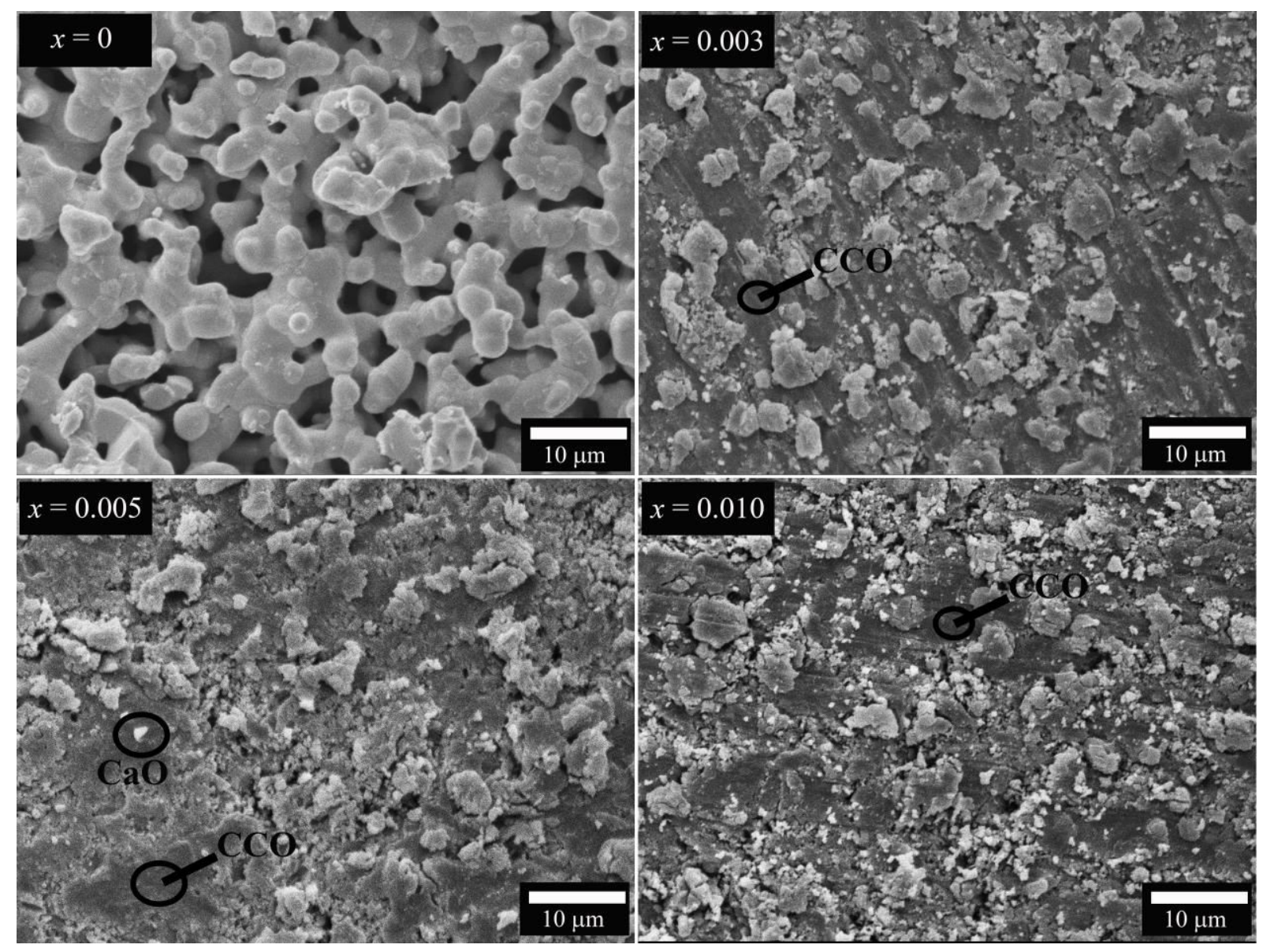


Fig. 2

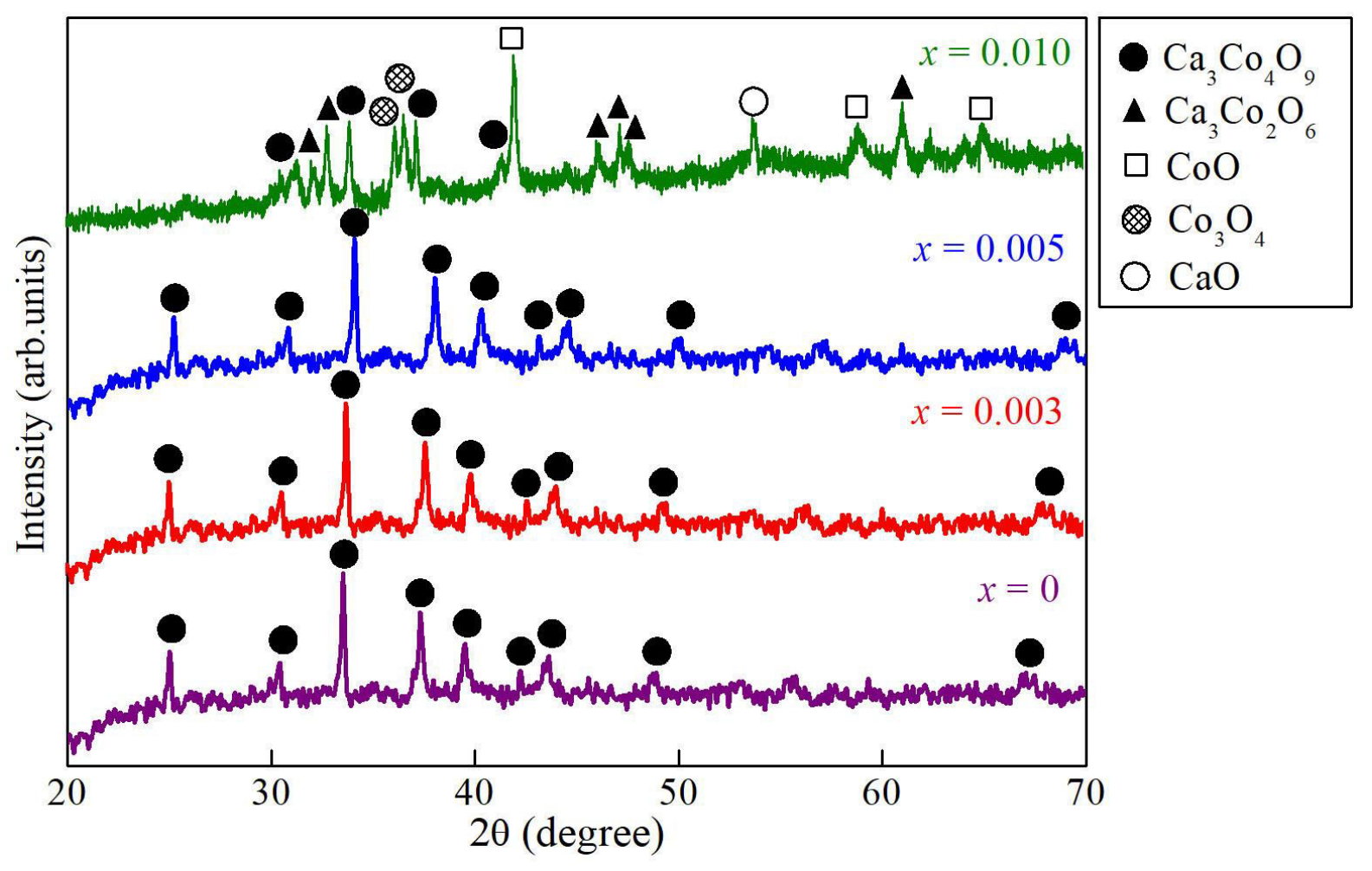


Fig. 3
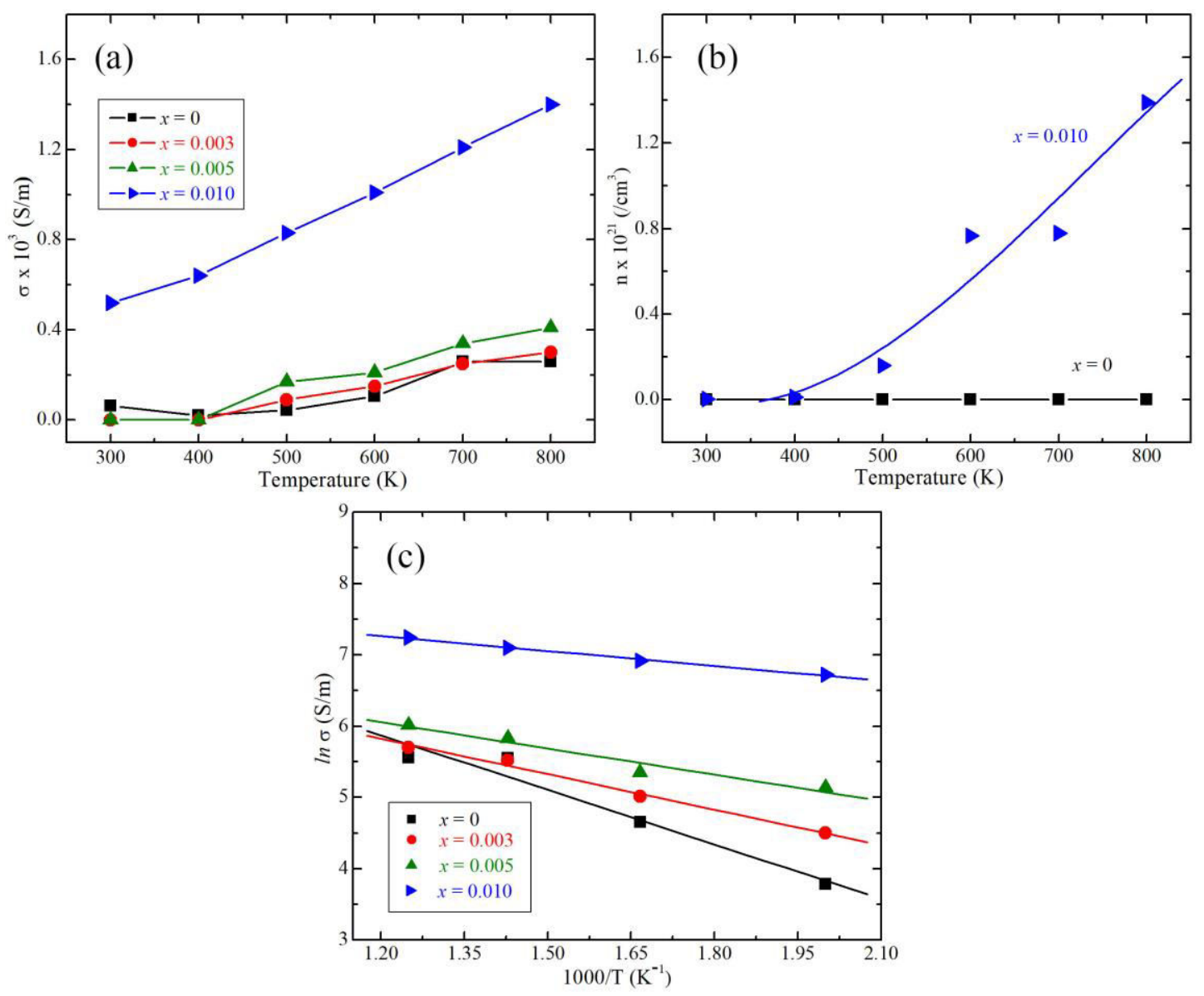
Fig. 4
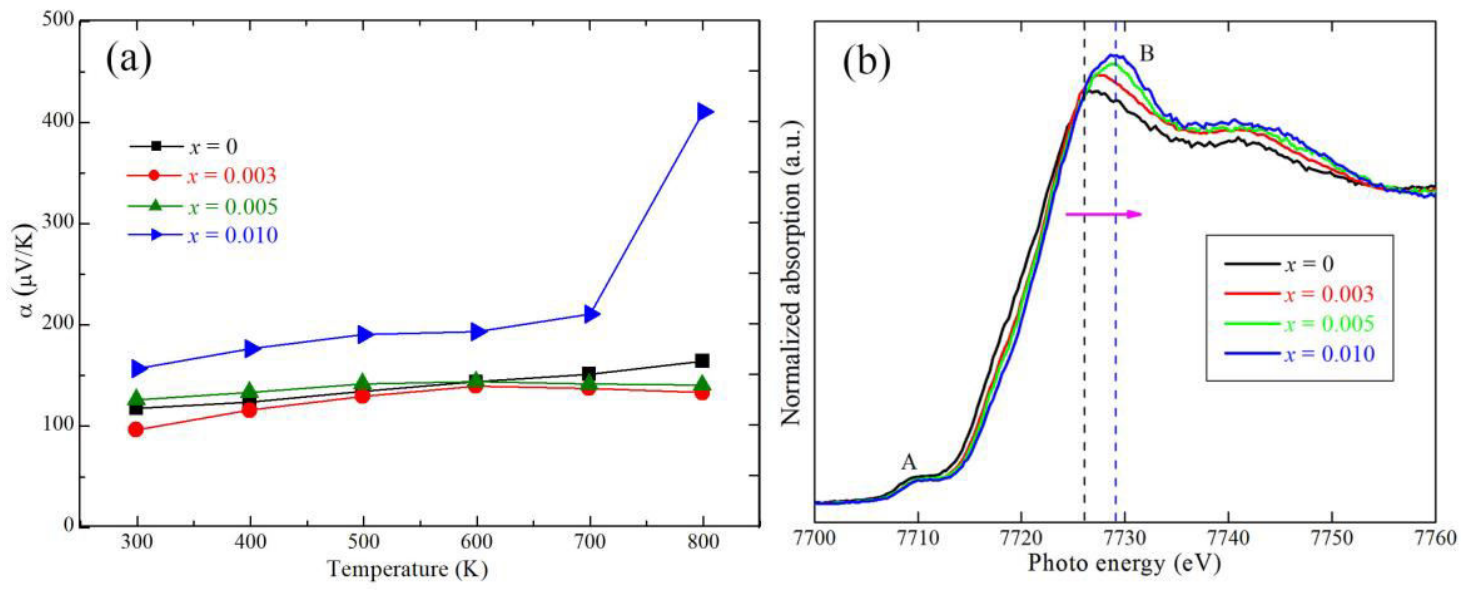
Fig. 5
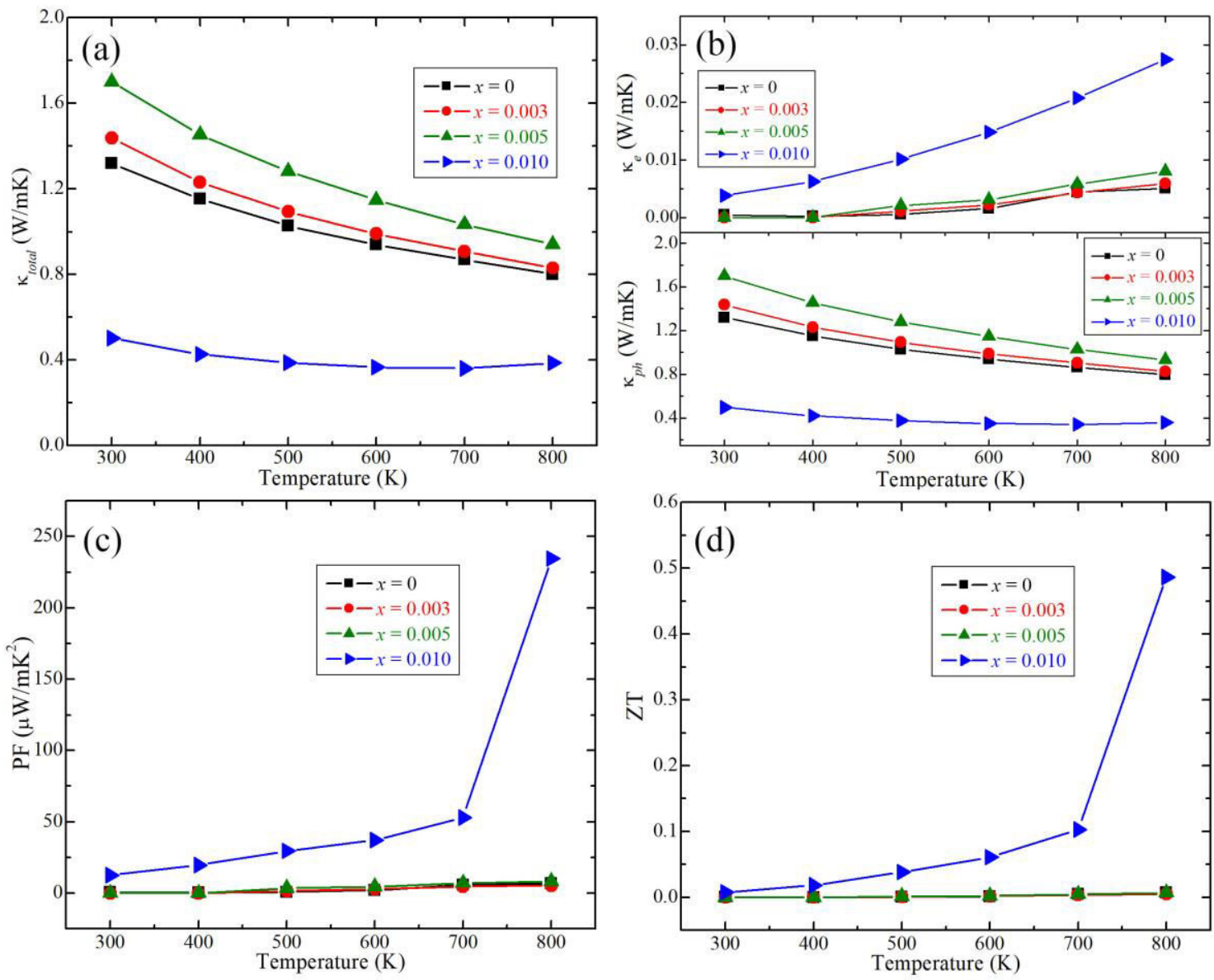


\section{Figure Captions}

Fig. 1 SEM images for all ceramics.

Fig. 2 XRD patterns in the 2-theta range of $20^{\circ}-70^{\circ}$ for all ceramics

Fig. 3 (a) is electrical conductivities as a function of temperature range from $300 \mathrm{~K}$ to $800 \mathrm{~K}$ for all ceramics, (b) is the charge carrier concentration as a function of the temperature of the composition $x=0$, and 0.010 , and (c) is Arrhenius's plots for all ceramics.

Fig. 4 (a) is the Seebeck coefficients as a function of temperature range from $300 \mathrm{~K}$ to $800 \mathrm{~K}$ for all ceramics, and (b) is XAS $K$-edges spectra of Co ions for all ceramics.

Fig. 5 (a) is thermal conductivities as a function of temperature range from $300 \mathrm{~K}$ to $800 \mathrm{~K}$ for all ceramics, (b) is phonon, and electronic thermal conductivities as a function of temperature for all ceramics, (c) is thermoelectric power factor as a function of temperature for all ceramics, and (d) is a figure of merit as a function of temperature for all ceramics. 
Table 1 Bulk and relative density values, and $E_{a}$ of all ceramics.

\begin{tabular}{lccc}
\hline BCZT content $(x)$ & Bulk density $\left(\mathrm{g} / \mathrm{cm}^{3}\right)$ & Relative density $(\%)$ & $E_{a}(\mathrm{eV})$ \\
\hline 0 & 4.22 & 90.1 & 0.220 \\
0.003 & 4.38 & 93.5 & 0.143 \\
0.005 & 4.43 & 94.7 & 0.106 \\
0.010 & 4.63 & 98.9 & 0.059 \\
\hline
\end{tabular}


